\title{
Molecular hydrogen as a new selective anti-oxidation therapeutic medical gas for clinical research
}

\author{
Jinqi Li, Yan Zang, Haihui Gu*, Baohua Qian* \\ Department of Transfusion Medicine, Shanghai Changhai Hospital, Second Military Medical University, Shanghai 200433, China.
}

\begin{abstract}
Hydrogen $\left(\mathrm{H}_{2}\right)$ is a colorless, odorless gas that can act as a reducing agent under certain circumstances. Previously considered physiologically an inert and nonfunctional molecule in mammalian cells, $\mathrm{H}_{2}$ largely went ignored until Nature Medicine revealed the antioxidant and cytoprotective effects of hydrogen gas in a focal stroke model. Reactive oxygen species (ROS) is generated inside the body throughout our daily lives as a by-product of the energy metabolism by oxidative phosphorylation, which can cause biofilm system damage and intracellular oxidative phosphorylation disorders. $\mathrm{H}_{2}$ reacts with highly reactive oxidants such as hydroxyl radical $(\bullet \mathrm{OH})$ and peroxynitrite $\left(\mathrm{ONOO}^{-}\right)$inside cells to improve ischemia reperfusion injury. In addition, hydrogen is a potent antiapoptotic and anti-inflammatory agent and can be used for potential medical applications in cells, tissues and organs. As a new antioxidant, hydrogen has the advantages of non-toxicity, easy diffusion and selective neutralization. This review makes a case for supporting hydrogen as a new antioxidant medicine for clinical applications. We also hope to provide a reference for the further study of hydrogen to preserve blood cells in transfusion medicine.
\end{abstract}

Keywords: hydrogen, reactive oxygen species, selective antioxidation

\section{INTRODUCTION}

Reactive oxygen species (ROS) is generated inside the body throughout our daily lives as a by-product of the energy metabolism by oxidative phosphorylation in every aerobic organism, including superoxide anion $\left(\mathrm{O}^{2-}\right)$, hydroxyl radical $(\bullet \mathrm{OH})$, hydroperoxide free radical $(\mathrm{HOO} \bullet)$, peroxynitrite $\left(\mathrm{ONOO}^{-}\right)$and so on. It is known to increase rapidly under certain types of physical exposure (such as ultraviolet or irradiation rays) or chemical exposure (such as aromatic hydrocarbons or pesticides). Usually, the body can limit damage caused

\footnotetext{
*Correspondence to: Baohua Qian, Department of Transfusion Medicine, Shanghai Changhai Hospital, Second Military Medical University, 168 Changhai Road, Shanghai 200433, China. E-mail: qianbh1963@163.com; Haihui Gu, Department of Transfusion Medicine, Shanghai Changhai Hospital, Second Military Medical University, 168 Changhai Road, Shanghai 200433, China. E-mail: haihuigu@126.com The authors have declared no conflict of interests.
}

by enzymes (such as superoxide dismutase). However, if ROS is produced excessively or the endogenous antioxidant capacity is diminished (exceeding the body's compensatory capacity), an imbalance between oxidation and antioxidation will occur and leading to pathological damage, known as oxidative stress. The main molecules of oxidative stress are the hydroxyl radical and peroxynitrite which can damage the body by direct action or triggering a free radical chain reaction ${ }^{[1]}$. Evidence has established strong links between oxidative stress and a wide variety of pathologies, including apoptosis, aging process, chronic inflammatory processes as well as malignant diseases ${ }^{[2]}$.

As a reducing agent, $\mathrm{H}_{2}$ can scavenge deleterious ROS by selectively reacting with ROS (such as hydroxyl radical or peroxynitrite) in vivo, meaning that $\mathrm{H}_{2}$ has preventive and therapeutic applications in alleviating oxidative stress related diseases. It's three main advantages in antioxidation: Firstly, $\mathrm{H}_{2}$ as a gas, 
has a very low molecular weight, causing a high biomembrane penetration and intracellular diffusion capability which enhances cell affinity ${ }^{[3]}$. Secondly, $\mathrm{H}_{2}$ can selectively scavenge the deleterious hydroxyl radical or peroxynitrite while preserving other important ROS (such as $\mathrm{H}_{2} \mathrm{O}_{2}, \mathrm{O}_{2}^{-}$or NO, etc.) for normal signaling regulation, therefore being superior to other antioxidants with a strong reductive activity (such as vitamin C) which ensures safe application ${ }^{[3]}$. Thirdly, $\mathrm{H}_{2}$ has no cytotoxicity (even at high concentrations) which avoids the increased risk of mortality associated with other gases ${ }^{[4]}$. Owing to its selective antioxidation and lack of adverse effects, $\mathrm{H}_{2}$ has promising potential for clinical therapeutic applications.

\section{MOLECULAR MECHANISM}

\section{Reduction of hydroxyl radical}

ROS serve a necessary function as signaling molecules that critically modulate the activation of the immune system and thus participate in body defense. The cells contain natural radical scavengers, which as long as they are enough to neutralize radiolysis products, and the DNA may be protected. Conversely, when radiolysis products exceed the amount of scavengers, they produce $\bullet \mathrm{OH}$, which directly affect the macromolecules in cells, resulting in DNA breakage, lipid peroxidation, protein denaturation and so on, after which radiation damage and cancer induction may occur ${ }^{[5]}$. In conclusion, $\bullet \mathrm{OH}$ are highly toxic to the body, and can cause a free radical chain reaction which transmits signals through the cell membrane to produce lipid peroxides and oxidative stress products. In supporting research, Ning et al. ${ }^{[6]}$ confirmed that $\mathrm{H}_{2}$ can reduce the expression of ROS and oxidative stress products. While Ohta ${ }^{[7]}$ demonstrated that $\mathrm{H}_{2}$ reacts with strong oxidants such as hydroxyl radicals within cells. $\mathrm{H}_{2}$ rapidly diffuses into tissues and cells, and it is mild enough neither to disturb metabolic redox reactions nor to affect the function of ROS in cellular signaling $^{[7]}$.

\section{Reduction of peroxynitrite}

The nitric oxide (NO) can readily react with $\mathrm{O}^{2-}$ to produce peroxynitrite, which participates as a mediator in the regulation of vascular tone, neurotransmission, and immunity, among other metabolic and cell signaling effects ${ }^{[8]}$. Although $\mathrm{H}_{2}$ can't scavenge peroxynitrite, it is able to diminish the toxicity of peroxynitrite $^{[9]}$. This is in accordance with previous studies, which established that $\mathrm{H}_{2}$ or hydrogen rich water can reduce the concentration of peroxynitrite in animal models ${ }^{[10-12]}$.

\section{Regulating endogenous signal pathway}

Oxidative stress impacts multiple signaling pathways, including the extracellular signal-regulated protein kinase (ERK) $1 / 2, \mathrm{NF}-\kappa \mathrm{B}$, as well as nuclear factor-erythroid-related factor 2 (Nrf2) pathways. Along with selectively scavenging $\bullet \mathrm{OH}, \mathrm{H}_{2}$ may alleviate oxidative stress-induced injury by targeting these pathways ${ }^{[13]}$. Ke et al. ${ }^{[14]}$ has shown that Nrf2 can inhibit the inflammatory response in liver transplanted mice and inhibit hepatocellular necrosis/ apoptosis through hypoxia-inducible factor(HIF)1 a factor. Kawamura ${ }^{[15]}$ demonstrated that inhaled $2 \% \mathrm{H}_{2}$ can ameliorate hyperoxic lung injury through the induction of Nrf2-dependent genes, such as heme oxygenase $(\mathrm{HO})-1$. In conclusion, $\mathrm{H}_{2}$ may play an antioxidant role by activating $\mathrm{Nrf2}$ and raising target protein expression.

\section{Indirect effects}

The body has many antioxidant enzymes, including superoxide dismutase (SOD), catalase (CAT) and glutathione peroxidase (glutathione peroxidase, GSH$\mathrm{Px})$. The study showed that $\mathrm{H}_{2}$ can reduce oxidative stress damage by increasing endogenous antioxidant enzyme activity ${ }^{[16]}$. In summary, $\mathrm{H}_{2}$ can not only directly react to reduce oxidative stress damage, but also can indirectly exert antioxidant effects by inducing the endogenous antioxidant system.

\section{METHODS OF INGESTING HYDROGEN}

\section{Inhalation of hydrogen}

A direct method of obtaining $\mathrm{H}_{2}$ therapeutically is through inhalation by using a ventilator circuit, facemask, or nasal cannula. Patients typically inhale $\mathrm{H}_{2}$ through a facemask, whereas in animal models, $\mathrm{H}_{2}$ is commonly obtained through a ventilator that provides $\mathrm{H}_{2}$ electrolyzed from water. Inhaled $\mathrm{H}_{2}$ acts rapidly and may be used to treat acute oxidative stress ${ }^{[7]}$. Moreover, $\mathrm{H}_{2}$ inhalation is a safe and effective method for patients with acute diseases ${ }^{[17]}$.

\section{Oral ingestion of hydrogen-rich water}

$\mathrm{H}_{2}$ can be dissolved in water up to $0.8 \mathrm{mmol} / \mathrm{L}$ ( 1.6 $\mathrm{mg} / \mathrm{L})$ under atmospheric pressure at room temperature without changing $\mathrm{pH} . \mathrm{H}_{2}$-rich water can be made by 3 methods: firstly, by infusing $\mathrm{H}_{2}$ into water under high pressure; secondly, by electrolyzing water; thirdly, though the reaction of magnesium metal or its hydride with water. However, $\mathrm{H}_{2}$ must be stored correctly as it easily penetrates glass or plastic containers in a short time, with aluminum containers being the 
preferred choice for its storage ${ }^{[18,19]}$. Currently, $\mathrm{H}_{2}$-rich water is distributed, and available for purchase.

\section{Injection of hydrogen-rich saline}

Although administering oral hydrogen-rich water is safe and convenient, controlling the concentration of $\mathrm{H}_{2}$ obtained can be difficult, as it evaporates over time and can be lost before absorption in the gastrointestinal tract. Thus, hydrogen-rich saline injection may be used to control $\mathrm{H}_{2}$ doses accurately ${ }^{[20,21]}$. Nagatani et al. ${ }^{[22]}$ indicated that $\mathrm{H}_{2}$ intravenous solution is safe for acute cerebral infarction, including patients treated with tissue-plasminogen activator.

\section{Direct diffusion of hydrogen}

$\mathrm{H}_{2}$ can easily penetrate the skin and is easily distributed via blood flow throughout the body, so taking a warm $\mathrm{H}_{2}$ bath is a method of incorporating $\mathrm{H}_{2}$ into the body in daily life. Actually, $\mathrm{H}_{2}$ bath powders are commercially available in Japan. Noda ${ }^{[23]}$ discovered that $\mathrm{H}_{2}$ delivery to cardiac grafts during cold preservation, efficiently ameliorated myocardial injury due to cold I/R. This new method for cold storage should be further developed for potential therapeutic during transplantation as well as operation.

\section{CLINICAL RESEARCH AND APPLICATION}

\section{Respiratory system}

\section{Lung ischemia-reperfusion injury}

Lung ischemia-reperfusion injury (LIRI) refers to the phenomenon that the lung has experienced a period of ischemia, and the ischemic damage is further aggravated after recovery blood perfusion. The mechanism of LIRI includes a significant involvement of ROS, intracellular calcium influx, endothelial cell injury, leukocyte sequestration and activation in the pulmonary circulation, activation of the complementary system, and the release of inflammatory mediators such as arachidonic acid metabolites ${ }^{[24]}$. Liu et al. ${ }^{[25]}$ examined the effects of lung inflation with $3 \% \mathrm{H}_{2}$ during the cold ischemia phase of lung graft function in rats. They also found that $\mathrm{H}_{2}$ has an antioxidant and anti-inflammatory influence on LIRI when it is inhaled by patients. The subsequent year, Meng et al. ${ }^{[26]}$ found that $\mathrm{H}_{2}$ combined with Carbon monoxide(CO) will enhance lung protection in LIRI models of rats.

\section{Hyperoxic acute lung injury}

Hyperoxic acute lung injury (HALI) refers to the damage to the lungs secondary to exposure to elevated oxygen partial pressure. It is a major clinical problem for patients undergoing supplemental oxygen therapy and has mostly been a concern in clinical practice with the development of deep diving ${ }^{[27]}$. ROS plays an important role in HALI as Sun's study ${ }^{[28]}$ found that $\mathrm{H}_{2}$ could reduce HALI, as well as oxidative stress. Later, Sun's team studied the mechanism of $\mathrm{H}_{2}$ in rats, finding that $\mathrm{H}_{2}$ could significantly reduce HALI by reducing lung edema and apoptosis, inhibiting the elevation of endoplasmic reticulum stress (ERS) and increasing SIRTl expression. This indicated that $\mathrm{H}_{2}$ reduced HALI related ERS and the mechanism may be associated with the upregulation of SIRTI.

\section{Acute lung injury}

Acute lung injury (ALI) is a complex clinical syndrome involved acute inflammation, microvascular damage, and increased pulmonary vascular and epithelial permeability, frequently resulting in acute respiratory failure culminating in fatal acute respiratory distress syndrome ${ }^{[29]}$. Ying ${ }^{[30]}$ reported in 2017 that hydrogen water attenuated ALI induced by oleic acid in rats and might protect against ALI through selective antioxidation and inhibiting inflammatory infiltration. Audi ${ }^{[31]}$ detected and tracked the antioxidant and antiapoptotic properties of $\mathrm{H}_{2}$ therapy in vivo in as early as $24 \mathrm{~h}$ after hyperoxia exposure. The result showed that $\mathrm{H}_{2}$ provided protection in rat models of ALI by inhalation. Also hydrogen saline may present a novel therapeutic approach for the treatment of ALI in Liu's study ${ }^{[32]}$, where it may aid in protecting against lung injury. While the underlying mechanism regarding the effect of hydrogen saline is unknown, $\mathrm{Du}^{[33]}$ supposed the mechanism may be associated with hydrogen inhibiting the release of pro-inflammatory cytokines, promoting anti-inflammatory cytokine release, and reducing oxidative damage.

\section{Nervous system}

\section{Intracerebral hemorrhage}

Intracerebral hemorrhage (ICH) is a devastating stroke subtype with high mortality and morbidity rates. At present, no specific medical therapy is available to treat $\mathrm{ICH}$. There is evidence to suggest that ROS damage the blood-brain barrier and increase brain injury. The $\bullet \mathrm{OH}$ and peroxynitrites are active ROS that react indiscriminately with nucleic acids, lipids, and proteins, resulting in DNA fragmentation, lipid peroxidation, and protein inactivation ${ }^{[34]}$. Takeuchi ${ }^{[35]}$ focused on the effects of medical treatment only using $\mathrm{H}_{2}$ (without surgery) on ICH. The results showed $\mathrm{H}_{2}$ depressed oxidative DNA damage in the brain, but unexpectedly had no beneficial effects on the brain edema, which means the mechanism of ROS in ICH may be more complicated than previ- 
ously believed. Therefore further studies are required to investigate whether $\mathrm{H}_{2}$ is effective for ICH.

\section{Neuronal ischemia-reperfusion (I/R) injury}

Acute neuronal injury during I/R has been attributed to loss of mitochondrial permeability transition coupled with mitochondrial dysfunction. Cui et al. ${ }^{[36]}$ showed that hydrogen rich saline was able to attenuate neuronal I/R injury, probably by selectively reducing cytotoxic oxygen radical function in rats. Moreover, Zhou $^{[37]}$ found the beneficial effects of hydrogen rich saline against spinal cord I/R injury by reducing oxidative stress.

\section{Neurodegenerative disorder}

Neurodegenerative disorders, including mild cognitive impairment (MCI) and dementia attributes to oxidative stress. Nishimaki ${ }^{[38]}$ assessed the effect of hydrogen rich saline on oxidative stress model mice and subjects with MCI. They found hydrogen rich water reduced oxidative stress markers and suppressed the decline of memory impairment and neurodegeneration. Dohi ${ }^{[39]}$ also found that hydrogen rich saline reverses neurodegenerative changes induced by traumatic brain injury.

\section{Carbon monoxide (CO) poisoning}

Neuronal injury caused by acute $\mathrm{CO}$ poisoning is partly free radical induced. In a model of $\mathrm{CO}$ induced poisoning, rats treated with $\mathrm{H}_{2}-$ rich saline significantly reduced the generation of ROS and subsequent lipid peroxidation in the nerve cell, improving the cognitive deficits. The mechanism of this protection may be related to reducing oxidative injury by affecting cells in vivo ${ }^{[40]}$.

\section{Cardiovascular system}

In heart failure induced by cardiac fibrosis attributing to sustained pressure overload, oxidative stress plays a significant role in cardiac remodeling and heart failure independently of etiological factors. Yang ${ }^{[41]}$ observed that $\mathrm{H}_{2}$ saline treatment improved interstitial fibrosis and cardiac function, decreased the level of ROS, the oxidative stress marker malondialdehyde(MDA) and the expression of nitrogen oxides(NOXs), while increasing the activity of the antioxidant enzyme SOD. These results indicate that $\mathrm{H}_{2}$ saline can improve cardiac function by reducing interstitial fibrosis through its antioxidative fuctions. In addition, $\mathrm{Wu}^{[42]}$ investigated the preventive effects of $\mathrm{H}_{2}$ treatment on doxorubicin-induced heart failure in rats. The results showed that the plasma level of oxidative stress markers were decreased in animals treated with $\mathrm{H}_{2}$ saline. Additionally, Shinbo ${ }^{[43]}$ con- cluded that breathing $\mathrm{H}_{2}$ plus $\mathrm{NO}$ may have beneficial effects for $\mathrm{I} / \mathrm{R}$ injury in murine heart.

\section{Digestive system}

Xia et al. ${ }^{[44]}$ reported that hydrogen-rich water significantly attenuated oxidative stress in chronic hepatitis B patients, but the effects of hydrogen-rich water on HBV DNA load and liver function has yet to be confirmed. In 2017 , Ikeda et al ${ }^{[45]}$ discovered that hydrogen-rich water can down-regulate the oxidative stress marker malondialdehyde(MDA), which prevented intestinal dysbiosis and could potentially be a new therapeutic strategy in cases of critical disease. Based on the observation that acetaminophen induces hepatotoxicity in blood and liver samples, Zhang ${ }^{[46]}$ discovered that hydrogen-rich water has significant therapeutic potential in inhibiting oxidative stress and promoting liver regeneration.

\section{Other systems}

In brief, $\mathrm{H}_{2}$ has promising preventive and therapeutic applications in various systems. For example, hydrogen-rich water is effective in preventing acute hearing loss due to transient cochlear ischemia ${ }^{[47]}$. It has also been found to visibly improve liver ischemiareperfusion injury ${ }^{[48]}$. In addition, hydrogen-containing gas $(1.3 \%$ hydrogen $+20.8 \%$ oxygen $+77.9 \%$ nitrogen) pre-inhaled can alleviate radiation-induced skin injury $^{[49]}$.

\section{Application in transfusion medicine}

In 2014, our team demonstrated that $\mathrm{H}_{2}$ had great efficacy and lack of adverse effects on platelet stor$\operatorname{age}^{[50]}$. During storage, platelets experience a series of transformations in morphology, function, as well as inhibition, known as platelet storage lesions. These injuries lead to platelet apoptosis, a weakening of clinical efficacy and in advanced cases the rendering of transfusions ineffective. We added hydrogen to platelets which were stored at $(22 \pm 2){ }^{\circ} \mathrm{C}$ and found hydrogen helped to maintain $\mathrm{pH}$ and reduce the expression of CD62p. Hydrogen gas may play an effective role in preserving platelets by inhibiting the denigration of platelets and maintaining the $\mathrm{pH}$ value in the plasma. While the mechanism has not been fully clarified, we suspect that it is related to antioxidation. Hydrogen is a promising agent for blood cell storage in transfusion medicine.

\section{EXPECTATION}

Hydrogen has wide application prospects in clinical research. The mechanism has not only antioxidant 
property, but also anti-inflammatory, anti apoptotic, anti allergic and the ability to promote the energy metabolism. These functions are coordinated together. However, research on the mechanism of hydrogen is still only superficial and the molecular target of antioxidation is still unknown. We hope that the target of hydrogen can be found not only to serve patients undergoing clinical procedures, but also for the preservation of blood products.

\section{Acknowledgments and funding}

This work was supported by grants of National Natural Science Foundation of China (81400152, 81570185), Shanghai Municipal Commission of Health and Family Planning Youth Project (20144Y0261) and Educational Research Project of the First Affiliated Hospital of Naval Military Medical University (CHJG 2017021).

\section{References}

[1] Liochev SI. Reactive oxygen species and the free radical theory of aging.Free Radic Biol Med,2013,60(10):1-4.

[2] Kim YW, Byzova TV. Oxidative stress in angiogenesis and vascular disease. Blood,2014,123(5):625-31.

[3] Kato S, Matsuoka D, Miwa N. Antioxidant activities of nano-bubble hydrogen-dissolved water assessed by ESR and 2,2'-bipyridyl methods.Mater Sci Eng C Mater Biol Appl, 2015,53:7-10.

[4] Ichihara M, Sobue S, Ito M, et al. Beneficial biological effects and the underlying mechanisms of molecular hydrogen - comprehensive review of 321 original articles. Med Gas Res,2015,5(1):12.

[5] Siezak J, Kura B, Frimmel K, et al. Preventive and therapeutic application of molecular hydrogen in situations with excessive production of free radicals.Physiol Res, 2016,65(S1):S11-28.

[6] Ning Y, Shang Y, Huang H, et al. Attenuation of cigarette smokeinduced airway mucus production by hydrogen-rich saline in rats.PLoS One,2013,8(12): e83429.

[7] Ohta S. Molecular hydrogen as a preventive and therapeutic medical gas: initiation, development and potential of hydrogen medicine.Pharmacol Th,2014,144(1):1.

[8] Radi R. Peroxynitrite, a stealthy biological oxidant. J Biol Chem,2013,288(37): 26464-72.

[9] Penders J, Kissner R, Koppenol WH. ONOOH does not react with $\mathrm{H} 2$ : Potential beneficial effects of $\mathrm{H} 2$ as an antioxidant by selective reaction with hydroxyl radicals and peroxynitrite.Free Radic Biol Med,2014,75(10):191-4.

[10] Shinbo T, Kokubo K, Sato Y, et al. Breathing nitric oxide plus hydrogen gas reduces ischemia-reperfusion injury and nitrotyrosine production in murine heart. Am J Physiol Heart Circ Physiol, 2013,305(4): H542-50.

[11] McCarty MF.Potential ghrelin-mediated benefits and risks of hydrogen water. Med Hypotheses,2015,84(4):350-5.

[12] Yokota T, Kamimura N, Igarashi T, et al. Protective effect of molecular hydrogen against oxidative stress caused by peroxynitrite derived from nitric oxide in rat retina. Clin Exp Ophthalmol, 2015,43(6):568-77.

[13] Xiao M, Zhu T, Wang T, et al. Hydrogen-rich saline reduces airway remodeling via inactivation of NF-kappaB in a murine model of asthma. Eur Rev Med Pharmacol Sci, 2013,17(8):1033-43.

[14] Ke B, Shen XD, Zhang Y, et al. KEAP1-NRF2 complex in ischenmia-induced hepatocellular damage of mouse liver transplants. J Hepatol, 2013,59(6):1200-7.

[15] Kawamura T, Wakabayashi N, Shigemura N, et al. Hydrogen gas reduces hyperoxic lung injury via the Nrf2 pathway in vivo.Am J Physiol Lung Cell Mol Physiol, 2013,304(10):646-56.

[16] Liu L, Xie K, Chen H, et al. Inhalation of hydrogen gas attenuates brain injury in mice with cecal ligation and puncture via inhibiting neuroinflammation, oxidative stress and neuronal apoptosis. Brain Res,2014,1589:7892.

[17] Ono H, Nishijima Y, Ohta S, et al. Hydrogen gas inhalation treatment in acute cerebral infarction: a randomized controlled clinical study on safety and neuroprotection. $J$ Stroke Cerebrovasc Dis, 2017,26(11):2587-94

[18] Wang T, Zhao L, Liu M, et al. Oral intake of hydrogenrich water ameliorated chlorpyrifos-induced neurotoxicity in rats. Toxicol Appl Pharmacol, 2014,280(1):169-76.

[19] Zhang J, Wu Q, Song S, et al. Effect of hydrogen-rich water on acute peritonitis of rat models. Int Immunopharmacol, 2014,21(1):94-101.

[20] Li G, Ji M, Sun X, et al. Effects of hydrogen-rich saline treatment on polymicrobial sepsis. J Surg Res, 2013,181(2):279-86.

[21] Zhai Y, Zhou X, Dai Q, et al. Hydrogen-rich saline ameliorates lung injury associated with cecal ligation and puncture-induced sepsis in rats.Exp Mol Pathol,2015,98(2):268-76.

[22] Nagatani K, Nawashiro H, Takeuchi S, et al. Safety of intravenous administration of hydrogen-enriched fluid in patients with acute cerebral ischemia: initial clinical studies. Med Gas Res,2013,3(1):13.

[23] Noda K, Shigemura N, TanakaY, et al. A novel method of preserving cardiac grafts using a hydrogen-rich water bath. J Heart Lung Transplant,2013,32(2):241-50.

[24] Renata SF, Cristiano FA. Oxidative stress and lung ischemia-reperfusion injury. Oxid Med Cell Longev,2015,2015(2):590987.

[25] Liu R, Fang X, Meng C, et al. Lung inflation with hydrogen during the cold ischemia phase decreases lung graft injury in rats.Exp Biol Med,2015,240(9):1214-22.

[26] Meng C, Ma L, Niu L, et al. Protection of donor lung inflation in the setting of cold ischemia against ischemiareperfusion injury with carbon monoxide, hydrogen, or both in rats. Life Sci,2016,151:199-206.

[27] Wen WL, Cui HH, Pei XZ, et al. Nitric oxide and hyperoxic acute lung injury.Med Gas Res,2016,6(2):85-95.

[28] Sun Q, Han W, Hu H, et al. Hydrogen alleviates hy- 
peroxic acute lung injury related endoplasmic reticulum stress in rats through upregulation of SIRT1.Free Radic Res,2017,51(6):622-32.

[29] Allen TC, Kurdowska A. Interleukin 8 and acute lung injury.Arch Pathol Lab Med,2014,138(2):266-9.

[30] Ying Y, Xu H, Yao M, et al. Protective effect of hydrogen-saturated saline on acute lung injury induced by oleic acid in rats.J Orthop Surg Res,2017,12(1):134.

[31] Audi SH, Jacobs ER, Zhang X, et al. Protection by inhaled hydrogen therapy in a rat model of acute lung injury can be tracked in vivo using molecular imaging. Shock,2017,48(4):467-76.

[32] Liu Y, Zhang J. Saturated hydrogen saline ameliorates lipopolysaccharide-induced acute lung injury by reducing excessive autophagy.Exp Ther Med,2017,13(6):2609-15.

[33] Du Z, Jia H, Liu J, et al. Effects of three hydrogenrich liquids on hemorrhagic shock in rats.J Surg Res,2015,193(1):377-82.

[34] Zhao H, Pan P, Yang Y, et al. Endogenous hydrogen sulphide attenuates NLRP3 inflammasome-mediated neuroinflammation by suppressing the P2X7 receptor after intracerebral haemorrhage in rats.J Neuroinflammation,2017,14(1):163.

[35] Takeuchi S, Nagatani K, Otani N, et al. Hydrogen does not exert neuroprotective effects or improve functional outcomes in rats after intracerebral hemorrhage. Turk Neurosurg,2016,26(6):854-9.

[36] Cui Y, Zhang H, Ji M, et al. Hydrogen-rich saline attenuates neuronal ischemia--reperfusion injury by protecting mitochondrial function in rats.J Surg Res,2014,192(2):564-72.

[37] Zhou L, Wang X, Xue W, et al. Beneficial effects of hydrogen-rich saline against spinal cord ischemia-reperfusion injury in rabbits.Brain Res,2013,1517(26):15060.

[38] Nishimaki K, Asada T, Ohsawa I, et al. Effects of molecular hydrogen assessed by an animal model and a randomized clinical study on mild cognitive impairment. Curr Alzheimer Res,2017, doi: 10.2174/15672050146661 71106145017.

[39] Dohi K, Kraemer BC, Erickson MA, et al. Molecular hydrogen in drinking water protects against neurodegenerative changes induced by traumatic brain injury.PLoS One,2014,9(9):e108034.
[40] Manaenko A, Lekic T, Ma Q, et al. Hydrogen inhalation ameliorated mast cell-mediated brain injury after intracerebral hemorrhage in mice. Crit Care Med,2013,41(5):1266-75.

[41] Yang J, Wu S, Zhu L, et al. Hydrogen-containing saline alleviates pressure overload-induced interstitial fibrosis and cardiac dysfunction in rats. $\mathrm{Mol} \mathrm{Med}$ Rep,2017,16(2):1771-8.

[42] Wu S, Zhu L, Yang J, et al. Hydrogen-containing saline attenuates doxorubicin-induced heart failure in rats. Pharmazie,2014,69(8):633-6.

[43] Shinbo T, Kokubo K, Sato Y, et al. Breathing nitric oxide plus hydrogen gas reduces ischemia-reperfusion injury and nitrotyrosine production in murine heart.Am J Physiol Heart Circ Physiol,2013, 305(4):542-50.

[44] Xia C, Liu W, Zeng D, et al. Effect of hydrogenrich water on oxidative stress, liver function, and viral load in patients with chronic hepatitis B.Clin Transl Sci,2013,6(5):372-5.

[45] Ikeda M, Shimizu K, Ogura H, et al. Hydrogen-rich saline regulates intestinal barrier dysfunction, dysbiosis and bacterial translocation in a murine model of sepsis. Shock,2017, doi: 10.1097/SHK.0000000000001098.

[46] Zhang JY, Song SD, Pang Q, et al. Hydrogen-rich water protects against acetaminophen-induced hepatotoxicity in mice.World J Gastroenterol,2015,21(14):4195-209.

[47] Ogawa H, Okada M, Shudou M, et al. Prevention of ischemia-induced hearing loss by intravenous administration of hydrogen-rich saline in gerbil.Neurosci Lett,2018,665:195-9.

[48] Li H, Bai G, Ge Y, et al. Hydrogen-rich saline protects against small-scale liver ischemia-reperfusion injury by inhibiting endoplasmic reticulum stress.Life Sci,2018,194:7-14.

[49] Watanabe S, Fujita M, Ishihara M, et al. Protective effect of inhalation of hydrogen gas on radiationinduced dermatitis and skin injury in rats.J Radtat Res,2014,55(6):1107-13.

[50] Haihui G, Yanxia Z, Meixian H, et al. The effects of adding saturated hydrogen gas to the platelets before platelet preservation. Chin J Blood Transfusion(In Chinese), 2014, 27(4):367-70.

(Received 20 February 2018, Revised 28 February 2018, Accepted 05 March 2018) 